\title{
Hidrogenación de m-Dinitrobenceno mediante el uso de catalizadores de platino soportados en silice, titania y alúmina.
}

MATERIALS ENGINEERING

\section{m-Dinitrobenzene hydrogenation using supported platinum catalysts over silica, titania and alumina}

\author{
Hugo A. Rojas*§, Gloria Borda*, María H. Brijaldo**, Jesús S. Valencia**, Patrício \\ Reyes***. \\ *Grupo de Catálisis. Universidad Pedagógica y Tecnológica de Colombia. Facultad de Ciencias. \\ ** Departamento de Química. Universidad Nacional de Colombia. \\ *** Laboratorio de Catálisis III. Universidad de Concepción
}

§hurojas@udec.cl,gborda98@hotmail.com,hbrijaldor@unal.edu.com,jsvalenciar@unal.edu.co, preyes@udec.cl.

(Recibido: Septiembre 21 de 2010 - Aceptado: Noviembre 9 de 2011 -Versión final: Mayo 08 de 2012)

\begin{abstract}
Resumen
Los catalizadores $\mathrm{Pt} / \mathrm{SiO} 2, \mathrm{Pt} / \mathrm{Al} 2 \mathrm{O} 3$ y $\mathrm{Pt} / \mathrm{TiO} 2$ se prepararon mediante impregnación húmeda, a una concentración del $1 \%$ en peso del metal. Los catalizadores resultantes se caracterizaron mediante análisis de difracción de rayos $\mathrm{X}$ (DRX), fisisorción de nitrógeno a $77 \mathrm{~K}$ y quimisorción de hidrógeno a $298 \mathrm{~K}$. Estos sistemas catalíticos se emplearon en la reacción de hidrogenación de m-dinitrobenceno para la obtención de m-feniléndiamina, una amina aromática de extensa aplicación en el campo de la química fina. Las reacciones se llevaron a cabo en un reactor tipo batch, a 0.82 MPa de presión de hidrógeno, $343 \mathrm{~K}$ y etanol como disolvente. Los productos de reacción se siguieron mediante cromatografía de gases. El catalizador Pt/TiO2 exhibió el mayor nivel de conversión de m-dinitrobenceno (91.5 \%) y selectividad hacia m-feniléndiamina (72.4\%). Este comportamiento se atribuyó a la presencia fuerte interacción entre el metal y el soporte.
\end{abstract}

Palabras Claves: amina aromática, m-dinitrobenceno, metal, selectividad.

\begin{abstract}
The $\mathrm{Pt} / \mathrm{SiO} 2, \mathrm{Pt} / \mathrm{A} 12 \mathrm{O} 3$ y $\mathrm{Pt} / \mathrm{TiO} 2$ catalysts through wet impregnation at $1 \%$ concentration in weigh metal were prepared. The resulting catalysts were characterized by X-ray diffraction (XRD), nitrogen physisorption (77 K) and hydrogen chemisorptions $(298 \mathrm{~K})$. Theses catalytic systems were employed in the reaction of m-dinitrobenzene hydrogenation toward $\mathrm{m}$-phenylenediamine. The m-phenylenediamine is aromatic amine used in fine chemicals. The reactions were carried out in batch reactor to hydrogen pressure of $0.82 \mathrm{MPa}, 343 \mathrm{~K}$, using ethanol as solvent. The products resulting were analyzed by gas chromatography. The results showed that $\mathrm{Pt} / \mathrm{TiO} 2$ catalyst had a high level of selectivity toward m-phenylenediamine (72.4\%) and level of conversion of m-dinitrobenzene $(91.5 \%)$. This behavior could be attributed to the strong metal-support interaction, as well as this catalyst is promising by production of m-phenylenediamine.
\end{abstract}

Keywords: aromatic amine, m-dinitrobenzene, metal, selectivity. 


\section{Introducción}

Los compuestos nitroaromáticos, constituidos por grupos nitro unidos directamente a un anillo bencénico, representan importantes intermediarios de reacción en la fabricación de numerosas moléculas orgánicas, Figueras (2001). Se utilizan principalmente en la síntesis de aminas aromáticas y aminas aromáticas sustituidas, cuya aplicación como precursores de polímeros, pigmentos, herbicidas, aditivos para cauchos o fármacos, entre otros, despierta un importante interés industrial, Studer et al. (2000).

En la reacción de hidrogenación de mdinitrobenceno (DNB) se obtiene por reducción del primer grupo nitro, el compuesto intermediario m-nitroanilina (MNA), luego se hidrogena el otro grupo nitro para obtener mfeniléndiamina (MPD). La reducción de $\mathrm{m}$ nitroanilina a m-feniléndiamina es difícil debido a la presencia del grupo nitro en posición meta, el cual desactiva el anillo bencénico, Belousov et al. (1988). Por esa razón es un desafío el desarrollo de catalizadores que puedan lograr una alta selectividad hacia m-feniléndiamina. La MPD es un importante material de partida de fibras de aramida, las cuales encuentran aplicación en los interiores de aeronaves donde es necesaria un fuerza tensil alta.

Diferentes métodos se han utilizado para sintetizar $\mathrm{m}$-feniléndiamina a partir de $\mathrm{m}$-dinitrobenceno, tales como; reducción de hierro con ácido clorhídrico (el proceso tradicional o proceso Bechamp), reducción electrolítica y la hidrogenación catalítica. La principal desventaja del proceso de reducción con hierro y ácido es la generación de gran cantidad de lodos de óxido férrico, lo cual plantea graves problemas de eliminación y por tanto es restringido en muchos países, mientras que el método de reducción con condensadores electrolíticos consume bastante energía, por lo tanto es antieconómico. Por otra parte, la hidrogenación catalítica de mdinitrobenceno en fase líquida es un método atractivo para aplicar tecnológicamente debido a su alto rendimiento de producción, relativas condiciones suaves de reacción y la reducción de la contaminación ambiental, Telkar et al. (2007).
La hidrogenación catalítica de $\mathrm{m}$-dinitrobeceno a $\mathrm{m}$-feniléndiamina se ha reportado con el uso de metales de transición como $\mathrm{Ni}$ y Fe, metales nobles soportados como $\mathrm{Pd}, \mathrm{y} \mathrm{Ru}$, Zhao et al. (2007). El Ni Raney usado para la hidrogenación de m-dinitrobenceno presenta baja conversión y selectividad hacia m-feniléndiamina. El Pd y Ru incrementan la conversión de m-dinitrobenceno sin embargo, la selectividad hacia mfeniléndiamina no supera el $40 \%$. El subproducto obtenido con una alta producción fue el compuesto intermediario, m-nitróanilina. Hasta ahora, poca información se conoce sobre hidrogenación de m-dinitrobenceno. Los catalizadores de platino son buenos candidatos debido a su gran poder de hidrogenación, ideal actividad catalítica con moléculas de interés en química fina y aplicación inocua al medio ambiente.

Para este estudio se utilizan diferentes óxidos ( $\mathrm{TiO} 2, \mathrm{~A} 12 \mathrm{O} 3$ y $\mathrm{SiO} 2$ ) que se caracterizan por interactuar de manera distinta con las fases soportadas. Como fase activa soportada se eligió el platino, uno de los metales nobles que mejores resultados produce en reacciones catalíticas, particularmente en las reacciones de hidrogenación selectiva. Este trabajo tiene como propósito estudiar la influencia del soporte en la reacción de hidrogenación de m-dinitrobenceno sobre catalizadores de Pt soportado en titania, alúmina y sílice, además se evalúa su incidencia en la actividad y selectividad hacia la obtención de la amina aromática, $\mathrm{m}$-feniléndiamina

\section{Metodología}

\subsection{Preparación de Catalizadores}

Los catalizadores de $\mathrm{Pt} / \mathrm{SiO} 2, \quad \mathrm{Pt} / \mathrm{TiO} 2$ y $\mathrm{Pt} / \mathrm{Al} 2 \mathrm{O} 3$ se prepararon por el método de impregnación húmeda de Sílice (Syloid 266 Grace Davison), Titania (Degusa P-25) y $\gamma$-alúmina $(\gamma-$ A12O3) respectivamente a $313 \mathrm{~K}$ con una cantidad adecuada de solución acuosa de $\mathrm{H} 2 \mathrm{PtCl} 6$ (Aldrich, 95\% de pureza) para obtener un precursor de catalizador al $1 \%$ en peso del metal, posteriormente se secó al vacio a $373 \mathrm{~K}$ por 6 horas, luego se calcinó a $673 \mathrm{~K}$ por 4 horas y se 
redujo en atmósfera de hidrógeno a temperatura de $773 \mathrm{~K}$ por dos horas. Se eligió ácido hexacloroplatinico (H2PtCl6) como precursor del metal, ya que en de acuerdo a algunas investigaciones publicadas, se caracteriza por presentar una adsorción fuerte sobre los soportes, por lo tanto se considera que en la etapa del secado no se afecta significativamente la distribución de platino obtenida durante la etapa de impregnación, Caballero (2002).

\subsection{Caracterización de Catalizadores}

Los catalizadores obtenidos fueron caracterizados en condiciones reducidas mediante fisisorción de nitrógeno a $77 \mathrm{~K}$ siguiendo el modelo de BET (Brunauer Emmet y Teller) para la determinación de área específica (SBET) y quimisorción de hidrógeno a $298 \mathrm{~K}$ en un equipo Micromeritics ASAP 2010, con el objeto de hallar la razón $\mathrm{H} / \mathrm{Pt}$ o dispersión (relación entre el número de átomos superficiales y numero de átomos metálicos totales) y presencia del efecto de interacción fuerte metalsoporte (SMSI).

Los análisis de difracción de rayos $\mathrm{X}$ (DRX) se efectuaron con el fin de obtener información de fases presentes en los catalizadores, estos se realizaron en un equipo Phillips PW 1140 con filtro de níquel y tiempo de exposición de 6 horas, con el uso de radiación de $\mathrm{K} \alpha \mathrm{Cu}$.

\subsection{Evaluación Catalítica}

Los estudios de evaluación catalítica permiten conocer el desempeño de catalizador en cuanto a actividad, selectividad y rendimiento, también son útiles para correlacionarlos con los resultados de caracterización y de esta forma explicar el comportamiento catalítico. Las condiciones iniciales de reacción fueron $343 \mathrm{~K}$ de temperatura de reacción y $0.82 \mathrm{MPa}$ de presión parcial de hidrógeno, empleando como disolvente etanol y una concentración de m-dinitrobenceno $0.1 \mathrm{M}$. Se utilizó un reactor tipo batch. La reacción se mantuvo a una agitación constante de $1000 \mathrm{rpm}$, esta condición asegura que la reacción no esté gobernada por efectos difusionales y de transporte de masa,. Se realizó seguimiento a la reacción por $7 \mathrm{~h}$.

\section{Resultados y discusión}

Los difractogramas de los catalizadores estudiados se muestran en la Figura 1. En ninguno de ellos se observa el pico de platino, debido al bajo contenido de metal $(1 \%)$, concentración que se encuentra cerca al límite de sensibilidad de la técnica, además, indica que el metal activo está bien distribuido en la superficie de los soportes. Se distinguen esencialmente las líneas del soporte en el caso de soportes cristalinos como la titania. Para el sistema $\mathrm{Pt} / \mathrm{TiO} 2$, se observan líneas de difracción, que comparadas con las tablas ASTM (American Society for Testing of Materiales) indican las presencia de las fases tetraédricas correspondientes a anatasa y rutilo. Este comportamiento también fue observado por Montes et al. (1996), para TiO2 (P-25), donde la mayor proporción en el pico anatasa comparada con rutilo, se debe a que en el tratamiento de calcinación a $673 \mathrm{~K}$ se favorece la formación de los cristales de anatasa. La titania, existe en tres formas cristalinas: rutilo, anatasa y brokita; el $\mathrm{TiO} 2$ que se usa normalmente como soporte para estudios catalíticos contiene las fases de anatasa y rutilo. Para este trabajo se utilizó Degussa P-25 TiO2 que contiene aproximadamente $85 \%$ de anatasa y $15 \%$ de rutilo a la temperatura de calcinación del soporte (673 K). El sistema Pt/SiO2 presenta un difractograma donde no se observan líneas de difracción correspondientes al soporte, lo cual indica que se trata de especies amorfas. Las señales del sistema $\mathrm{Pt} / \mathrm{A} 12 \mathrm{O} 3$ son anchas y difusas. Se observaron picos de difracción a $2 \theta ; 31.7^{\circ}$, $37.8^{\circ}, 45.3^{\circ}$ y $66.8^{\circ}$ características de la $\gamma$ - $\mathrm{Al} 2 \mathrm{O} 3$.

Los resultados de caracterización de área superficial (SBET) y relación $\mathrm{H} / \mathrm{Pt}$, se muestran en la Tabla 1. Se observó que el catalizador con mayor área superficial corresponde a $\mathrm{Pt} / \mathrm{SiO} 2$, esto concuerda con los resultados de difracción de rayos $X$, ya que los materiales amorfos presentan mayores áreas superficiales, Hoffman et al. (2002). Es notable observar que no se presentó variación significativa entre los valores obtenidos de área superficial para los catalizadores soportados en titania y alúmina, comparados con los valores de área superficial únicamente del soporte (óxido), situación que indica que la impregnación con platino no afecta el valor de SBET.

La razón H/Pt es mayor en sistemas que no sufren 


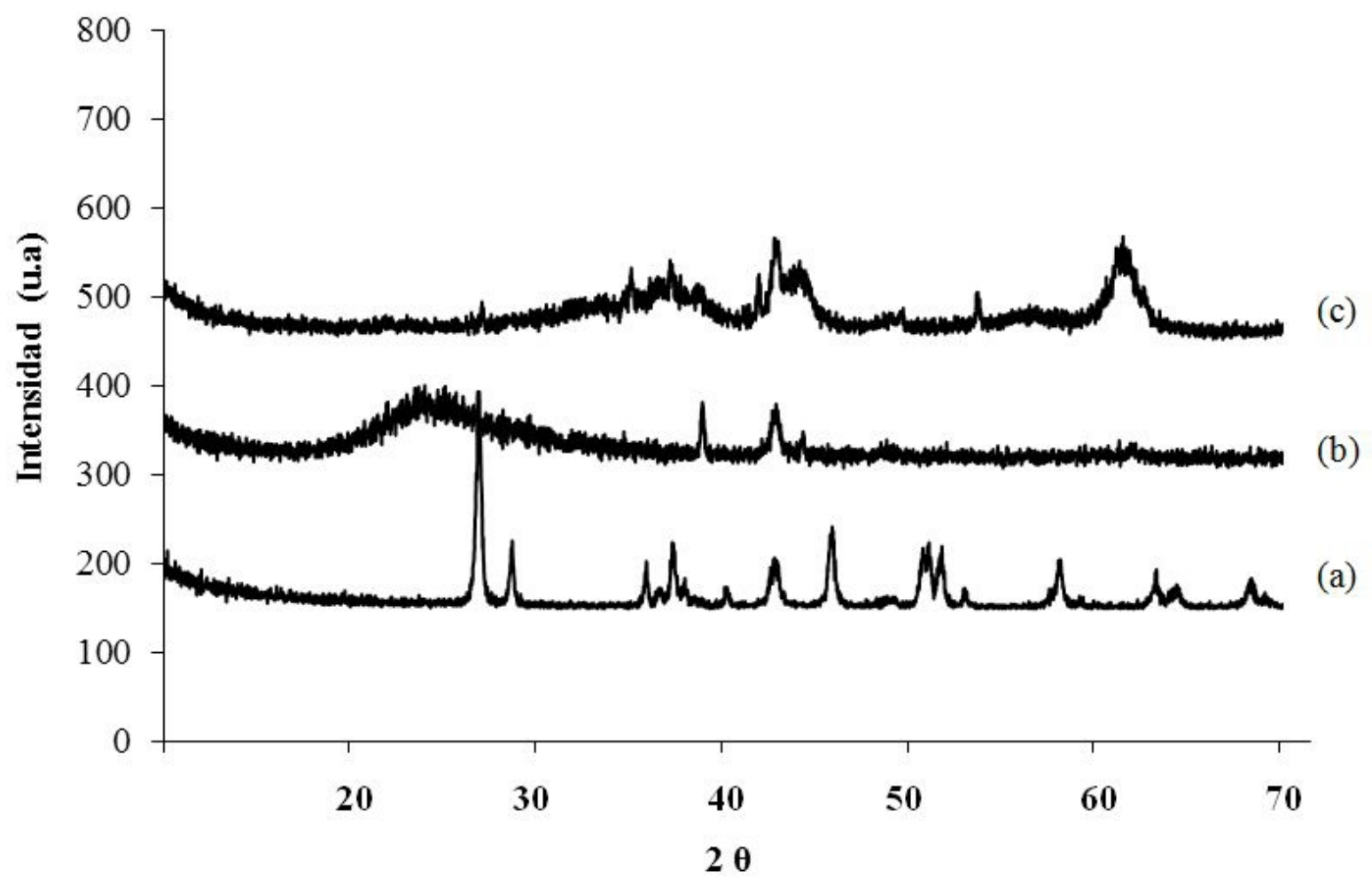

Figura 1 Difractogramas de rayos X para catalizadores de platino (1\%) soportado. a) $\mathrm{Pt} / \mathrm{TiO} 2$; b). $\mathrm{Pt} / \mathrm{SiO} 2$; c). Pt/Al2O3

Tabla 1. Superficie Específica (SBET) y Razón H/Pt para los catalizadores de Pt soportado.

\begin{tabular}{ccc}
\hline Catalizador & $\mathrm{S}_{\mathrm{BET}}\left(\mathrm{m}^{2} \mathrm{~g}^{-1}\right)$ & $\mathrm{H} / \mathrm{Pt}$ \\
\hline $\mathrm{Pt} / \mathrm{TiO}_{2}$ & 54 & 0.16 \\
$\mathrm{Pt} / \mathrm{SiO}_{2}$ & 278 & 0.20 \\
$\mathrm{Pt} / \mathrm{Al}_{2} \mathrm{O}_{3}$ & 189 & 0.85 \\
$\mathrm{TiO}_{2}$ & 54 & - \\
$\mathrm{SiO}_{2}$ & 279 & - \\
$\mathrm{Al}_{2} \mathrm{O}_{3}$ & 188 & - \\
\hline
\end{tabular}


reducción parcial del soporte, como en el caso de $\mathrm{Pt} / \mathrm{A} 12 \mathrm{O} 3$ y $\mathrm{Pt} / \mathrm{SiO} 2$. En el catalizador $\mathrm{Pt} / \mathrm{SiO} 2$, la la razón $\mathrm{H} / \mathrm{Pt}$ es relativamente menor, que en el sistema Pt/Al2O3, situación que se explica considerando que la sílice es un soporte muy ácido, su punto isoeléctrico es del orden de 1-2, por lo tanto la interacción con el precursor no se ve muy favorecida como es el caso de la alúmina y en consecuencia la dispersión metálica es menor. En el catalizador $\mathrm{Pt} / \mathrm{SiO} 2$ hay que tener en cuenta la naturaleza del precursor metálico empleado en su preparación, que fue $\mathrm{H} 2 \mathrm{PtCl6}$, por lo tanto las especies (PtCl6)2- provenientes del precursor son de naturaleza aniónica y la superficie del soporte por encima del punto isoeléctrico $(>2)$ se encuentra cargada negativamente, estas circunstancias hacen que no exista un enlace fuerte entre el metal y el soporte. Además, en la etapa de calcinación puede ocurrir procesos de sinterización del metal, ya que este estado depende fundamentalmente de la naturaleza de la interacción metal-soporte, ejemplo de ello son las fuerzas relativas metalmetal, versus las energías de enlace metal-soporte. Metales depositados en óxidos irreducibles (SiO2), la fuerza de enlace metal-metal es significativamente más grande que la del enlace metal-soporte, conduciendo a una interacción metal-soporte relativamente débil y fácil sinterización térmica.

Para el catalizador de Pt soportado en $\mathrm{TiO} 2$ la situación es diferente, y el cambio en la razón $\mathrm{H} / \mathrm{Pt}$ no puede atribuirse a cambios en dispersión, sino que se atribuye principalmente a la decoración del cristal metálico por especies del soporte parcialmente reducido (TiOx), a temperaturas cerca de $773 \mathrm{~K}$, fenómeno característico del efecto interacción fuerte metal soporte (SMSI), Reyes et al. (2007). Estas especies tienen alguna movilidad y pueden migrar desde la interfase metal-soporte, produciendo un cubrimiento parcial de la partícula metálica, lo que hace disminuir la capacidad de quimisorber hidrógeno y por ello la razón $\mathrm{H} / \mathrm{Pt}$ es menor.

La Tabla 2, resume los resultados de TOF o Actividad por Sitio (Turn Over Frequency), actividad inicial expresada en $\mu \mathrm{mol} / \mathrm{s}$.gcat a $10 \%$ de conversión y nivel de conversión de $m$ dinitrobenceno a 7 horas de reacción. Los valores de TOF de los catalizadores estudiados cambian entre sí, se observa que el sistema $\mathrm{Pt} / \mathrm{TiO}_{2}$ presenta el mayor valor $\left(2.12 \mathrm{~s}^{-1}\right)$. Puede explicarse este resultado como consecuencia del fenómeno SMSI, en donde por efecto de la migración de especies del soporte se crean sitios específicos más activos para la hidrogenación de grupos nitro. Los catalizadores $\mathrm{Pt} / \mathrm{Al}_{2} \mathrm{O}_{3}$ y $\mathrm{Pt} / \mathrm{SiO}_{2}$, presentan menores valores de TOF, estos sistemas no son reducibles, lo que sugiere que no se crean sitios activos en posiciones adecuadas para permitir la reacción química.

La evolución del nivel de conversión con el tiempo de reacción durante la hidrogenación del $m$-dinitrobenceno a $343 \mathrm{~K}$ y $0.82 \mathrm{MPa}$, en los sistemas catalíticos estudiados, indica que

Tabla 2. Resultados de TOF (Turn Over Frequency) $\left(\mathrm{s}^{-1}\right)$, actividad inicial expresada en $\mu \mathrm{mol} / \mathrm{s}$.gcat a $10 \%$ de conversión, así como el nivel de conversión de m-dinitrobenceno a 7 horas de reacción.

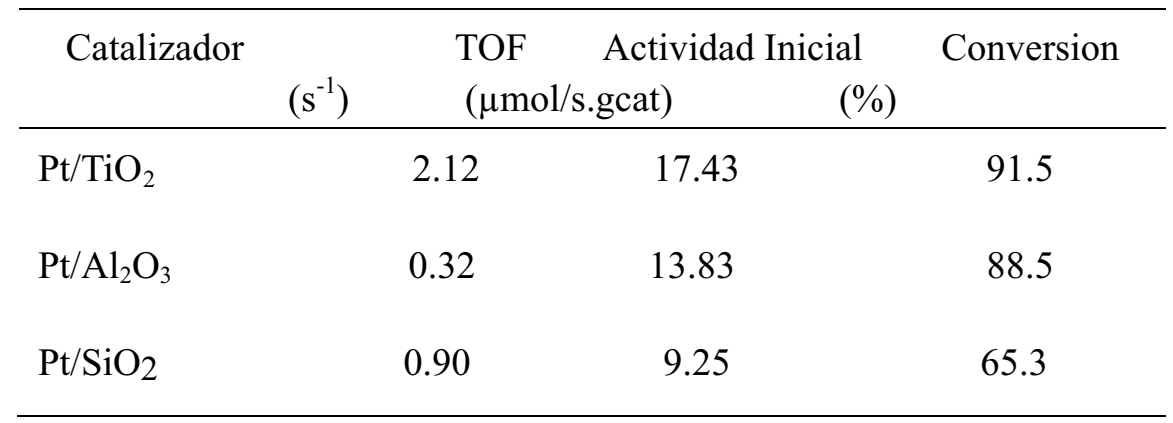


aumenta progresivamente a medida que transcurre el tiempo de reacción, Figura 2. Se observa que el mayor nivel de conversión lo presenta el catalizador $\mathrm{Pt} / \mathrm{TiO}_{2}$, con $91.5 \%$ seguido del sistema $\mathrm{Pt} / \mathrm{Al}_{2} \mathrm{O}_{3}$, mientras que en el catalizador $\mathrm{Pt} / \mathrm{SiO}_{2}$, tan solo se obtuvo $65.3 \%$ de conversión a 7 horas de reacción. Estos resultados, se explican para el catalizador $\mathrm{Pt} / \mathrm{TiO}_{2}$, porque posee además de un gran número de sitios de hidrogenación de las partículas de metal, sitios especiales de hidrogenación originados por el efecto SMSI.

En la reacción de hidrogenación de $m$ dinitrobenceno se detectaron como productos; $m$ nitroanilina y $m$-feniléndiamina (Tabla 3 ). Los catalizadores $\mathrm{Pt} / \mathrm{TiO}_{2}$ y $\mathrm{Pt} / \mathrm{Al}_{2} \mathrm{O}_{3}$ exhiben la formación de $m$-feniléndiamina $(72.4 \%$ y $15 \%$ respectivamente), mientras que en el sistema $\mathrm{Pt} / \mathrm{SiO}_{2}$ la selectividad es mayor del $99.9 \%$ hacia $m$-nitroanilina y no se observa presencia de la amina aromática. En la Figura 3 se muestra la formación de $m$-feniléndiamina a través del tiempo; se observa que con el uso del catalizador de $\mathrm{Pt} / \mathrm{TiO}_{2}$ la producción de la amina aromática empieza a 1 hora de iniciada la reacción, mientras que en el catalizador de $\mathrm{Pt} / \mathrm{Al}_{2} \mathrm{O}_{3}$ se detecta la presencia de $m$-feniléndiamina, 4 horas después. Estos resultados afirman que el catalizador $\mathrm{Pt} / \mathrm{TiO}_{2}$, es un sistema promisorio para llevar a efecto esta reacción y el soporte parcialmente reducible otorga gran selectividad para la obtención de MPD. Por otro lado, con el catalizador $\mathrm{Pt} / \mathrm{Al}_{2} \mathrm{O}_{3}$, si bien la producción de $m$ nitroanilina es relativamente alta, la producción de $m$-feniléndiamina es baja. Se explica este resultado probablemente por ausencia de reducción del soporte; como se indica por sus valores de dispersión (Tabla 1), el número de sitios hidrogenantes es alto, por eso el valor de actividad inicial es elevada (Tabla 2), pero difícilmente la molécula de $m$-nitroanilina encuentra sitios selectivos para formar $m$-feniléndiamina.

En la Figura 4 se observa el comportamiento del reactivo y productos a través del tiempo en la hidrogenación de $m$-dinitrobenceno para el catalizador $\mathrm{Pt} / \mathrm{TiO}_{2}$. Se observa que la concentración de $m$-dinitrobenceno decrece rápidamente con el tiempo de reacción, la concentración de $m$-nitroanilina aumenta hasta alcanzar una concentración máxima, y posteriormente disminuye, mientras que la $m$ feniléndiamina aumenta durante el transcurso de la reacción. Esta conducta es típica de una reacción consecutiva. Los anteriores resultados concuerdan con los obtenidos por Cárdenas et al. (2009), donde al hidrogenar $m$-dinitrobenceno obtuvo inicialmente $m$-nitroanilina como intermediario, la cual fue convertida al producto final, $m$ feniléndiamina. Finalmente, como se mencionó anteriormente el sistema $\mathrm{Pt} / \mathrm{TiO}_{2}$, presentó los mejores resultados de conversión y selectividad hacia $m$-feniléndiamina, debido a la creación de sitios metal-Ti en la interfase metal-soporte y a la existencia de la decoración de las partículas de $\mathrm{Pt}$ por especies del soporte parcialmente reducidas $\left(\mathrm{TiO}_{\mathrm{x}}\right)$, las cuales se generaron en la activación del catalizador a $773 \mathrm{~K}$. La presencia de este fenómeno contribuye favorablemente a la reducción de compuestos nitroaromáticos, como se ha demostrado en varios estudios reportados en la literatura, Corma et al., (2007), al emplear catalizadores de $\mathrm{Au} / \mathrm{TiO}_{2}$ en la hidrogenación de 3nitroestireno y nitrobenceno. Ellos también demostraron que la decoración del metal activo $(\mathrm{Pt}$, $\mathrm{Ru}$ y $\mathrm{Ni}$ ) por especies del soporte parcialmente reducidas $\left(\mathrm{TiO}_{\mathrm{x}}\right)$, resultó en una producción elevada de la amina aromática correspondiente.

Sin embargo, en el catalizador de $\mathrm{Pt} / \mathrm{SiO}_{2}$, se observó que una vez reducido el primer grupo nitro no hay posterior presencia de $m$-feniléndiamina. Este sistema no resultó efectivo para hidrogenar el grupo nitro en posición meta. Como se ha explicado en este catalizador incide la ausencia de reducción del soporte y la afinidad del metal por el soporte. Esta situación no permitió obtener valores de selectividad razonables que contribuyan a la formación de la amina aromática como producto final de la reacción. 


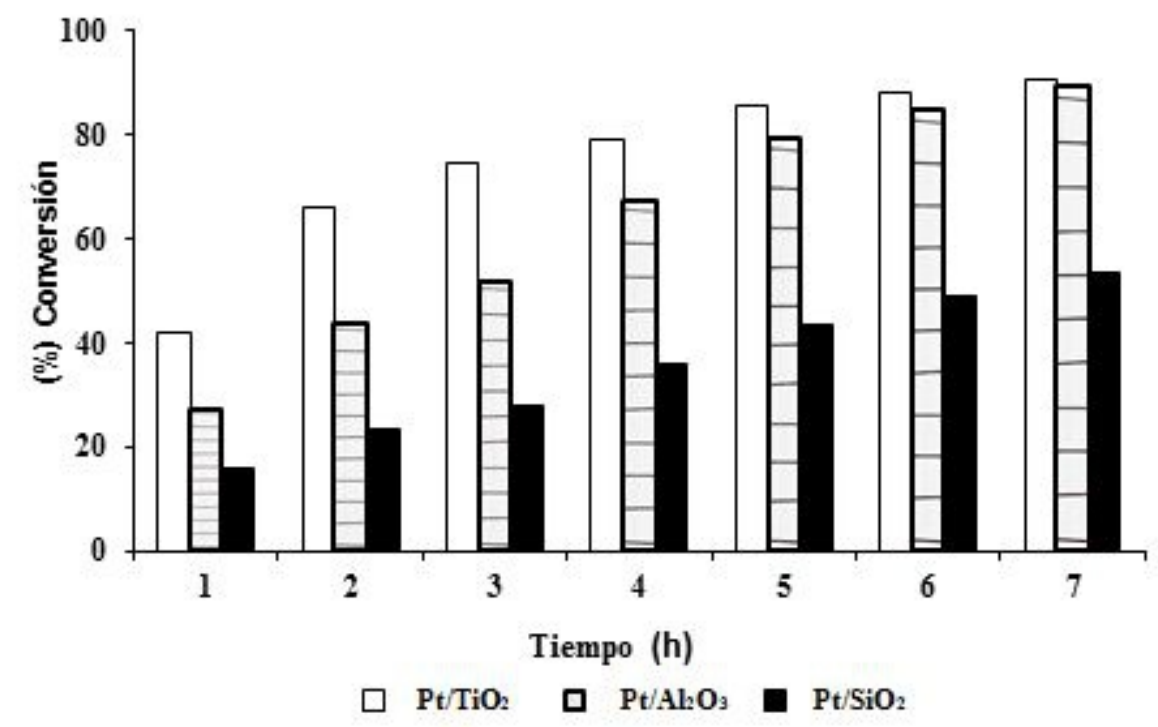

Figura 2. Nivel de conversión vs. tiempo en la hidrogenación de m-dinitrobenceno a $343 \mathrm{~K}$ y 0.82 MPa con catalizadores de platino soportado.

Tabla 3. Resultados de selectividad hacia m-nitroanilina y m-feniléndiamina a 7 horas de reacción.

\begin{tabular}{|c|c|c|}
\hline Productos & Catalizador & $\begin{array}{l}\text { Selectividad } \\
(\%)\end{array}$ \\
\hline \multirow[t]{3}{*}{$\mathrm{NH}_{2}$} & $\mathrm{Pt} / \mathrm{SiO}_{2}$ & $>99.9$ \\
\hline & $\mathrm{Pt} / \mathrm{Al}_{2} \mathrm{O}_{3}$ & 84.3 \\
\hline & $\mathrm{Pt} / \mathrm{TiO}_{2}$ & 27.5 \\
\hline
\end{tabular}

$m$-nitroanilina<smiles>Nc1cccc(N)c1</smiles>

$\mathrm{Pt} / \mathrm{SiO}_{2}$

15.7

$m$-feniléndiamina

$\mathrm{Pt} / \mathrm{Al}_{2} \mathrm{O}_{3}$

72.4

$\mathrm{Pt} / \mathrm{TiO}_{2}$ 


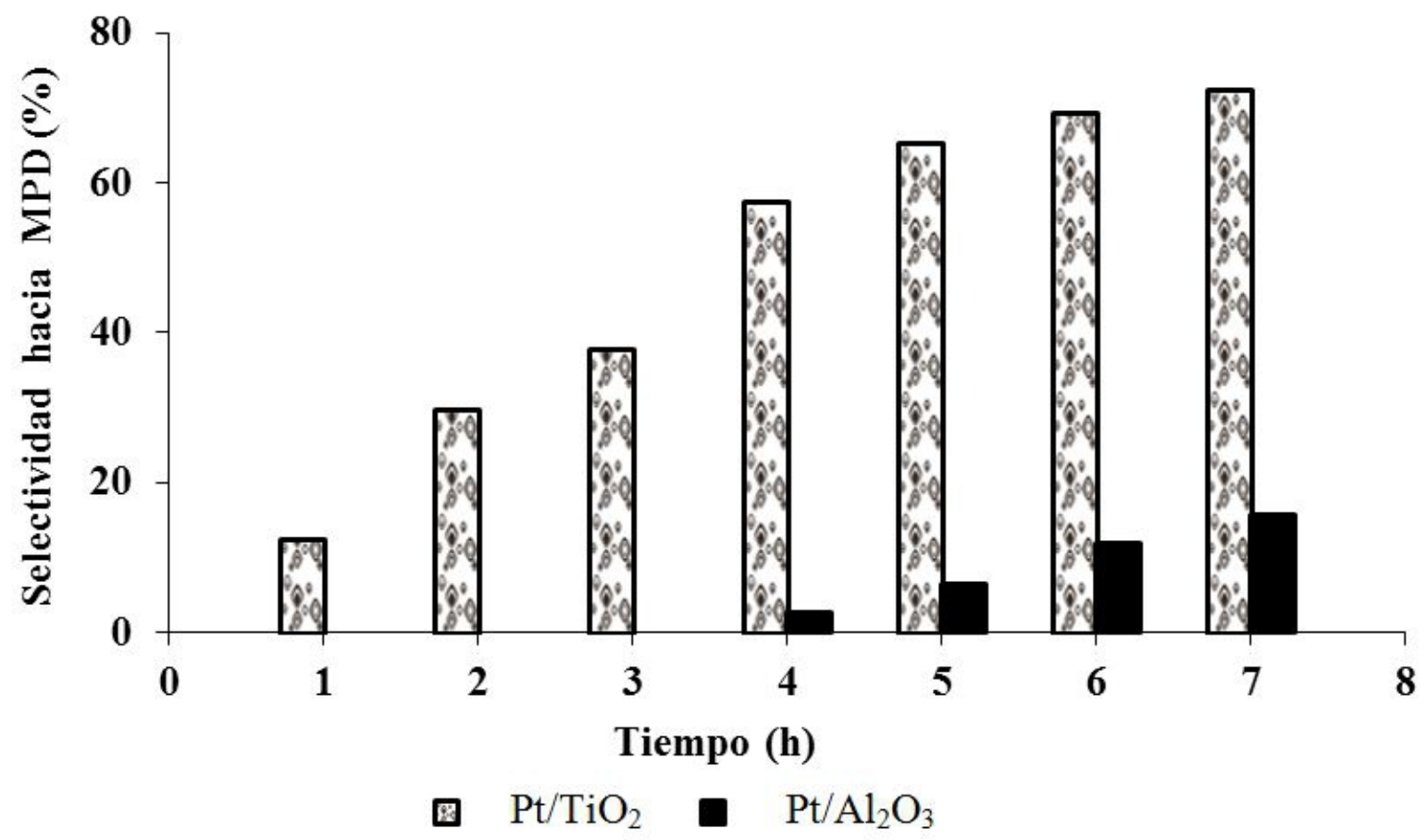

Figura 3. Nivel de selectividad hacia m-feniléndiamina en los catalizadores $\mathrm{Pt} / \mathrm{TiO} \mathrm{O}_{2}$ y $\mathrm{Pt} / \mathrm{Al}_{2} \mathrm{O}_{3}$ en el transcurso de la reacción.

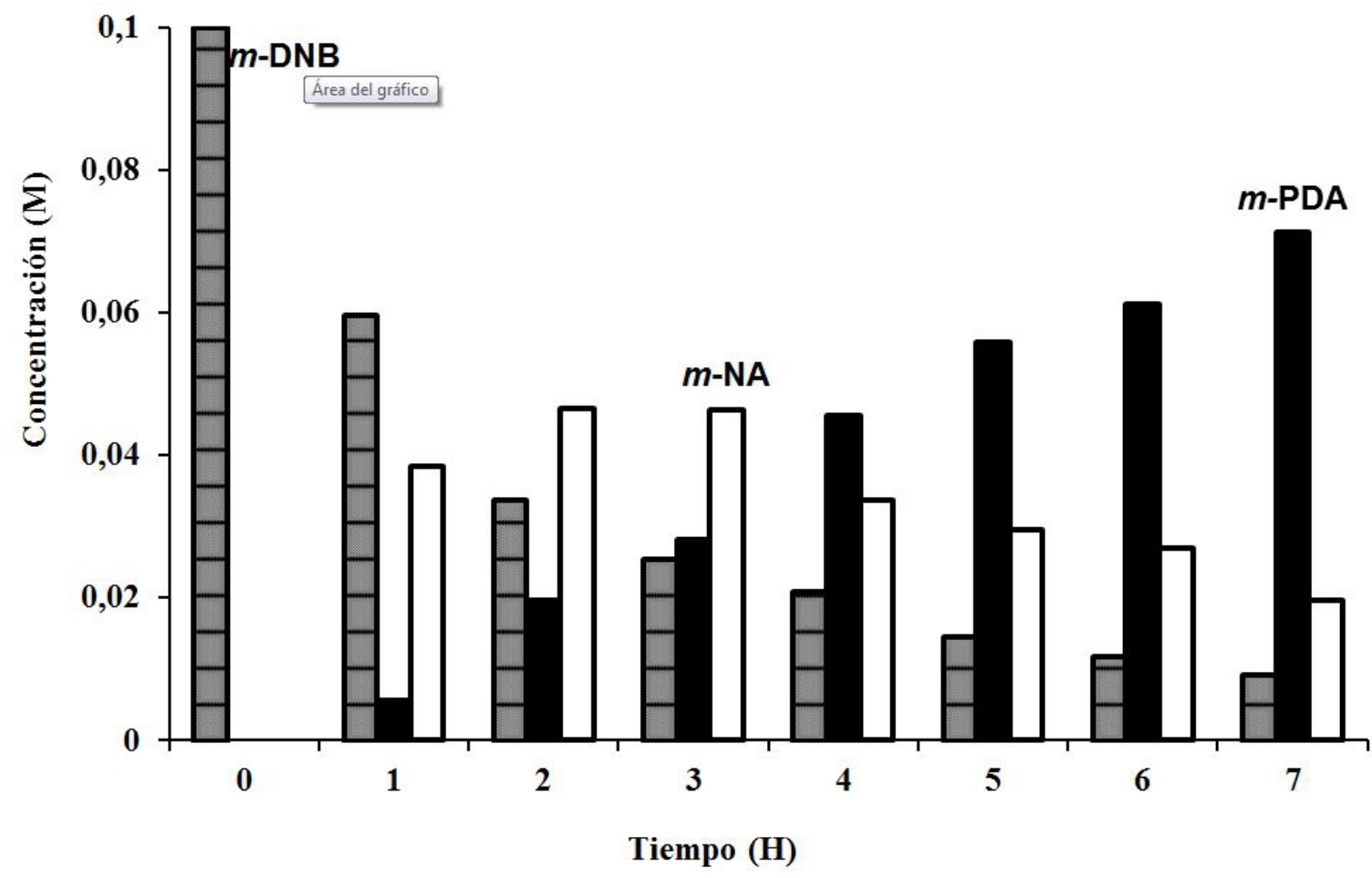

Figura 4. Concentraciones de reactivos y productos en el transcurso de la reacción con el catalizador Pt/TiO . 


\section{Conclusiones}

Los resultados indican que la formación de $m$ feniléndiamina, el cual es el producto de interés, se produce mediante reducción consecutiva de los grupos nitro del $m$-dinitrobenceno y cuyo principal intermediario es la $m$-nitroanilina.

Se obtuvieron catalizadores promisorios en la hidrogenación de nitroaromáticos sustituidos a partir de metales poco estudiados en este tipo de reacciones, siendo los sistemas de $\mathrm{Pt} / \mathrm{TiO}_{2}$ y $\mathrm{Pt} / \mathrm{Al}_{2} \mathrm{O}_{3}$ los que mejor comportamiento presentaron en la hidrogenación de $m$ dinitrobenceno.

En términos generales, la reacción de $m$ dinitrobenceno es fuertemente afectado por las propiedades físico-químicas de los soportes; la presencia de soportes que experimenten reducción y que a su vez interactué con la fase activa, son parámetros comunes en los catalizadores activos y selectivos para la formación de aminas aromáticas.

\section{Agradecimientos}

Los autores agradecen a la DIN-UPTC, por el apoyo financiero para el desarrollo del proyecto SGI 683. A RECIEND COMPANY por el suministro de titania $\left(\mathrm{TiO}_{2} \mathrm{P}-25\right)$, Lote 41680882698.

\section{Referencias bibliográficas}

Belousov, V., Palchevskaya, T., \& Bogutskaya, L. (1988). Influence of substituents on hydrogenation of nitro groups in aromatic hydrocarbons catalyzed by rhenium thiocomplexes. Reaction Kinetic Catalysis Letter 36 (2), 369-374.
Caballero L. (2002) Introducción a la catálisis heterogénea: Universidad Nacional de Colombia.

Cárdenas F., Gómez S., Idriss H., \& Keane M. (2009) Gold particle size effects in the gas-phase hydrogenation of $m$-dinitrobenzene over $\mathrm{Au} / \mathrm{TiO}_{2}$. Journal of Catalysis 268 (2) 223-234.

Corma A., Boronat M., Concepción P., González S., Illas F., Serna P. (2007) A molecular mechanism for the chemoselective hydrogenation of substituted nitroaromatics with nanoparticles of gold on $\mathrm{TiO}_{2}$ catalysts: A cooperative effect between gold and the support. Journal the American Chemical Society 129 (51) 16230-16237.

Figueras F., \& Coq B. (2001) Hydrogenation and hydrfogenolysis of nitro, nitroso, azo, asoxy and other nitrogen compounds on palladium. Journal Molecular Catalysis. A: Chemical. 173 (2) 223230.

Hoffman, H., Staudt, P., Costa, T., \& Benvenutti, E. (2002). FTIR Study of the electronic metalsupport interactions on platinum disperse on silica modified with titania. Surface and Interface Analysis 33 (8), 631-634.

Montes, F., Getton, P., Vong, W., \& Sermon, A. (1997). Titania on silica a comparison of sol gel routes y traditional methods. Journal of sol-gel. Revision Science and Technology 8 (1), 131-137.

Reyes, P., Borda, G., Rojas, H., Murcia, J., Fierro, J.L., \& Oportus, M. (2007). Hydrogenation of Citral on $\mathrm{Ir} / \mathrm{SiO}_{2}$ catalysts. Effect of the Addition of $\mathrm{Nb}_{2} \mathrm{O}_{5}$ on surface and catalytic properties. Reaction Kinetic Catalysis Letter. 92 (2), 369376.

Studer, M., Neto, S., \& Blaser H. (2000). Modulating the hydroxylamine accumulation in the hydrogenation of substituted nitroarenes using 
vanadium-promoted RNi catalysts. Topics in catalysis. 13 (3), 205-2012.

Telkar, M., Nadgeri, J. Rode, C., \& Chaudhari, R. (2005). Role of a co-metal in bimetallic Ni-Pt catalyst for hydrogenation of $m$-dinitrobenzene to $m$-phenylenediamine. Applied Catalysis A; General 295(1), 23-30.

Zhao, S., Huading, L., \& Zhou, Y. (2007). Selective hydrogenation of $m$-dinitrobenzene to $m$-nitroaniline catalyzed by $\mathrm{PVP}-\mathrm{Ru} / \mathrm{Al}_{2} \mathrm{O}_{3}$. Catalysis Communications 8 (9), 1305-1309. 